\title{
Human pluripotent stem cell (PSC)-derived mesenchymal stem cells (MSCs) show potent neurogenic capacity which is enhanced with cytoskeletal rearrangement
}

\author{
Kai-Yen Peng ${ }^{1,2}$, Yu-Wei Lee ${ }^{2}$, Pei-Ju Hsu ${ }^{2}$, Hsiu-Huan Wang ${ }^{2}$, Yun Wang ${ }^{3}$, \\ Jun-Yang Liou' ${ }^{2}$, Shan-Hui Hsu ${ }^{4}$, Kenneth K. Wu ${ }^{5}$, B. Linju Yen² \\ ${ }^{1}$ Department of Life Science, National Central University, Jhongli, Taiwan \\ ${ }^{2}$ Regenerative Medicine Research Group, Institute of Cellular and System Medicine, National Health Research Institutes \\ (NHRI), Zhunan, Taiwan \\ ${ }^{3}$ Center for Neuropsychiatric Research, NHRI, Zhunan, Taiwan \\ ${ }^{4}$ Institute of Polymer Science and Engineering, National Taiwan University, Taipei, Taiwan \\ ${ }^{5}$ Graduate Institute of Basic Medical Sciences, China Medical University, Taichung, Taiwan \\ Correspondence to: B. Linju Yen, email: blyen@nhri.org.tw \\ Keywords: mesenchymal stem cells (MSCS), human pluripotent stem cells (PSC), human embryonic stem cells (ESCs), \\ induced pluripotent stem cells (iPS), Rho A kinase (ROCK) \\ Received: April 07, 2016 \\ Accepted: May 23, 2016 \\ Published: June 11, 2016
}

\section{ABSTRACT}

Mesenchymal stem cells (MSCs) are paraxial mesodermal progenitors with potent immunomodulatory properties. Reports also indicate that MSCs can undergo neurallike differentiation, offering hope for use in neurodegenerative diseases. However, ex vivo expansion of these rare somatic stem cells for clinical use leads to cellular senescence. A newer source of MSCs derived from human pluripotent stem cells (PSC) can offer the 'best-of-both-worlds' scenario, abrogating the concern of teratoma formation while preserving PSC proliferative capacity. PSC-derived MSCs (PSC-MSCs) also represent MSCs at the earliest developmental stage, and we found that these MSCs harbor stronger neuro-differentiation capacity than post-natal MSCs. PSC-MSCs express higher levels of neural stem cell (NSC)-related genes and transcription factors than adult bone marrow MSCs at baseline, and rapidly differentiate into neural-like cells when cultured in either standard neurogenic differentiation medium (NDM) or when the cytoskeletal modulator RhoA kinase (ROCK) is inhibited. Interestingly, when NDM is combined with ROCK inhibition, PSC-MSCs undergo further commitment, acquiring characteristics of post-mitotic neurons including nuclear condensation, extensive dendritic growth, and neuron-restricted marker expression including NeuN, $\beta$-III-tubulin and Doublecortin. Our data demonstrates that PSC-MSCs have potent capacity to undergo neural differentiation and also implicate the important role of the cytoskeleton in neural lineage commitment.

\section{INTRODUCTION}

The discovery of a stem cell population in the adult brain has opened the possibility of using such tissuespecific stem cells for therapy in neuro-degenerative and ischemic diseases, including Parkinson's disease and stroke [1]. As exciting as these findings are, the rarity and difficulty in procurement of such neural stem cells (NSCs) are significant obstacles to prevalence use of these stem cells [2]. Thus, there is continued interest in finding more accessible sources of human stem cells for application in neurological diseases.

A number of studies have shown that mesenchymal stem cells (MSCs) harbor neural differentiation potential and have therapeutic relevance for treatment of related diseases [3]. Multilineage MSCs were first isolated from the bone marrow (BM) and have subsequently been found in various post-natal tissues [4, 5]. In addition to trilineage paraxial mesodermal differentiation capacity, MSCs have significant immunomodulatory properties, making 
these adult stem cells highly versatile for broad clinical application [6]. However, these rare post-natal stem cells often require vigorous ex vivo expansion to reach the volumes necessary for therapeutic use, and this can result in replicative senescence and functional decline [7]. To overcome this issue, MSCs have been derived from human pluripotent stem cells (PSC) including human embryonic stem cells (ESCs) and induced pluripotent stem cells (iPS), and these PSC-MSCs exhibit similar differentiation capacity and surface marker profile to BM-MSCs [8-10]. The differentiation of PSC to a somatic stem cell type such as MSCs allows for clinical use, since worries of teratoma formation is abrogated when PSCs lose pluripotency [11]. Moreover, PSC-MSCs have the added advantage of being derived from a renewable source since PSCs can theoretically be expanded indefinitely, unlike BM-MSCs and other tissue-specific MSCs which must be continually isolated from primary tissue [12]. Being derived directly from PSC, PSC-MSCs are also at a much earlier stage developmentally than adult BM-MSCs, and can possibly have broader differentiation capacity as has been reported for fetal-stage MSCs [13].

Traditionally, induction of neural differentiation has involved the use of biochemical factors in serumfree conditions [14]. Recent studies, however, show that cytoskeletal rearrangement can efficiently induce a neural-like phenotype in MSCs $[15,16]$. In this report, we found that PSC-MSCs express higher NSCs-related genes at baseline, and can efficiently differentiate into neuron-restricted progenitors (NRPs) with inhibition of Rho-associated protein kinases (ROCKs), key molecules in modulation of the cytoskeleton [17]. Our data implicate the use of PSC-MSCs in application towards neuro-related diseases, as well as the importance of the cytoskeleton in neural lineage commitment.

\section{RESULTS}

\section{PSC-MSCs express higher levels of neural stem cell (NSC)-associated genes at baseline than BM-MSCs}

To assess the neurogenic potential of human PSCMSCs, including human ESCs-derived MSCs (ES-MSCs) and iPS-derived MSCs (iPS-MSCs), we performed real-time PCR to detect baseline expression of Nestin, an intermediate filament, and Musashi, a RNA-binding protein, which are neural stem cell (NSC)-associated genes [18]. We found that at baseline, PSC-MSCs express significantly higher levels of Nestin and Musashi than adult BM-MSCs (Figure 1A). ES-MSCs (12.3-fold), iPSMSC-1 (11.9-fold), and iPS-MSC-2 (12.8-fold) express Nestin at a level that is on average over 10 -fold higher than BM-MSCs. The expression levels of Musashi were up to 30.0-fold higher in ES-MSCs (17.6-fold), iPS-MSCs-1 (25.9-fold), and iPS-MSC-2 (30.0-fold) compared to
BM-MSCs. We then performed neural differentiation using standard neurogenic differentiation medium (NDM), which consist of serum-free medium with addition of retinoic acid (RA) [14]. After culturing in NDM for two days, BM-MSCs and PSC-MSCs acquired a neural-like morphology with elongated cytoplasmic processes and numerous dendrite-like processes (Figure 1B). NDM also increased the expression of Nestin, Musashi, and MAP2 (microtubule-associated protein 2) - a critical protein involved in neural differentiation [19] — in all MSCs, with a more significant increase in PSCs-MSCs compared to BMMSCs (Figure 1C). These results suggest PSC-MSCs may have a higher neurogenic potential and are more sensitive to neurogenic differentiation signals than BM-MSCs.

\section{PSC-MSCs acquired an early-stage neural cell phenotype through inhibition of Rho a kinase- myosin II pathway}

Cytoskeletal rearrangement in post-natal MSCs has been shown to direct neural lineage commitment through RhoA kinase (ROCK), one of the most important mediators in cytoskeletal dynamics $[15,16]$. We sought to ascertain whether cytoskeletal rearrangement in PSCMSCs could also lead to neural lineage commitment. To address this question, we treated PSC-MSCs cultured in complete medium with Y-27632, a selective inhibitor of ROCK, then assessed for protein expression of neural lineage markers by immunofluorescent (IF) staining. Addition of Y-27632 in complete medium not only promoted a neuron-like morphology but also increased Nestin and MAP2 expression in PSC-MSCs to a similar extent compared to culturing in NDM (Figure 2A). It has been reported that the actions of ROCK inhibition in neural differentiation of MSCs involve myosin II, one of its downstream targets [20]. Correspondingly, when PSCMSCs are treated with blebbistatin, a specific inhibitor of myosin II ATPase, the morphological changes seen with ROCK inhibition are recapitulated $[15,21]$. To assess cytoskeletal dynamics during PSC-MSC differentiation, we stained for F-actin and found that PSC-MSCs treated with Y-27632 and blebbistatin show actin-rich extensions at the leading edge (Figure 2B), a characteristic of axonal and dendritic growth during neuronal development [22]. In contrast, in untreated PSC-MSCs, F-actin remained cytoplasmic. Thus, inhibition of the ROCK-myosin II axis induces neural lineage commitment in PSC-MSCS.

\section{ROCK inhibition of iPS-MSCs cultured in NDM induce further neural commitment}

Given the strong baseline expression of NSCrelated genes and response to cytoskeletal manipulation, we were interested whether PSC-MSCs could further undergo differentiation into more committed neural lineage cells. NSCs are known to further differentiate 

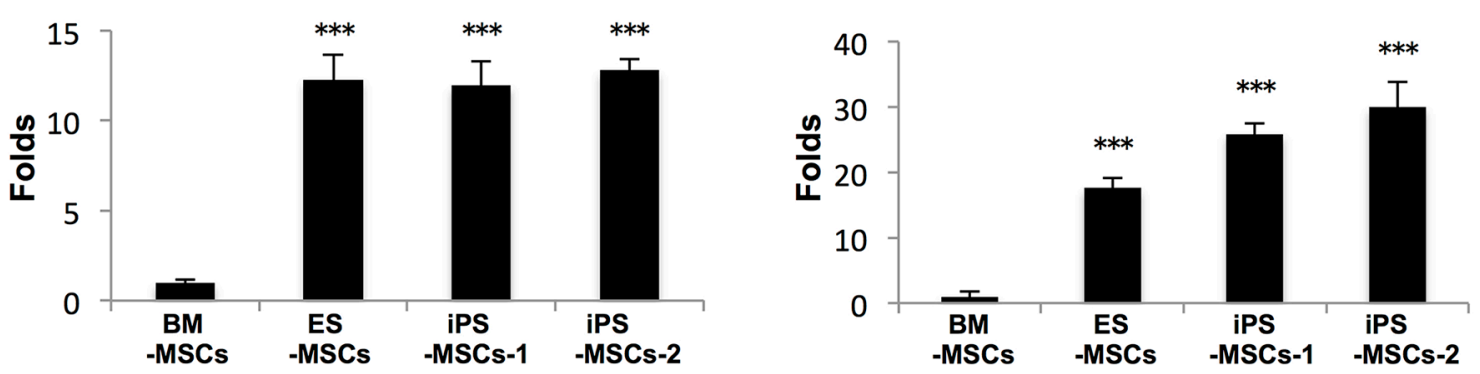

B

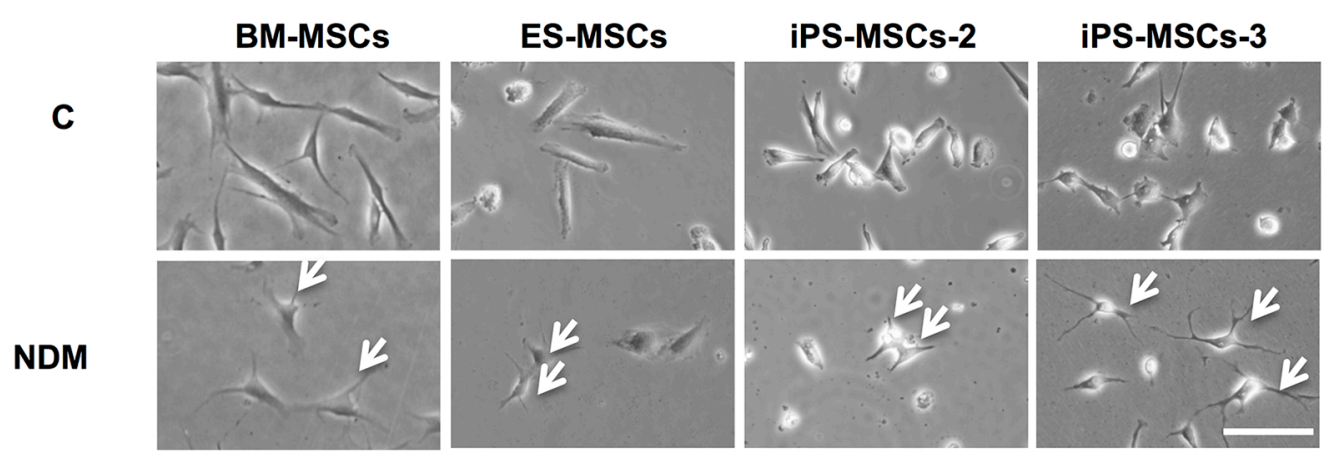

C
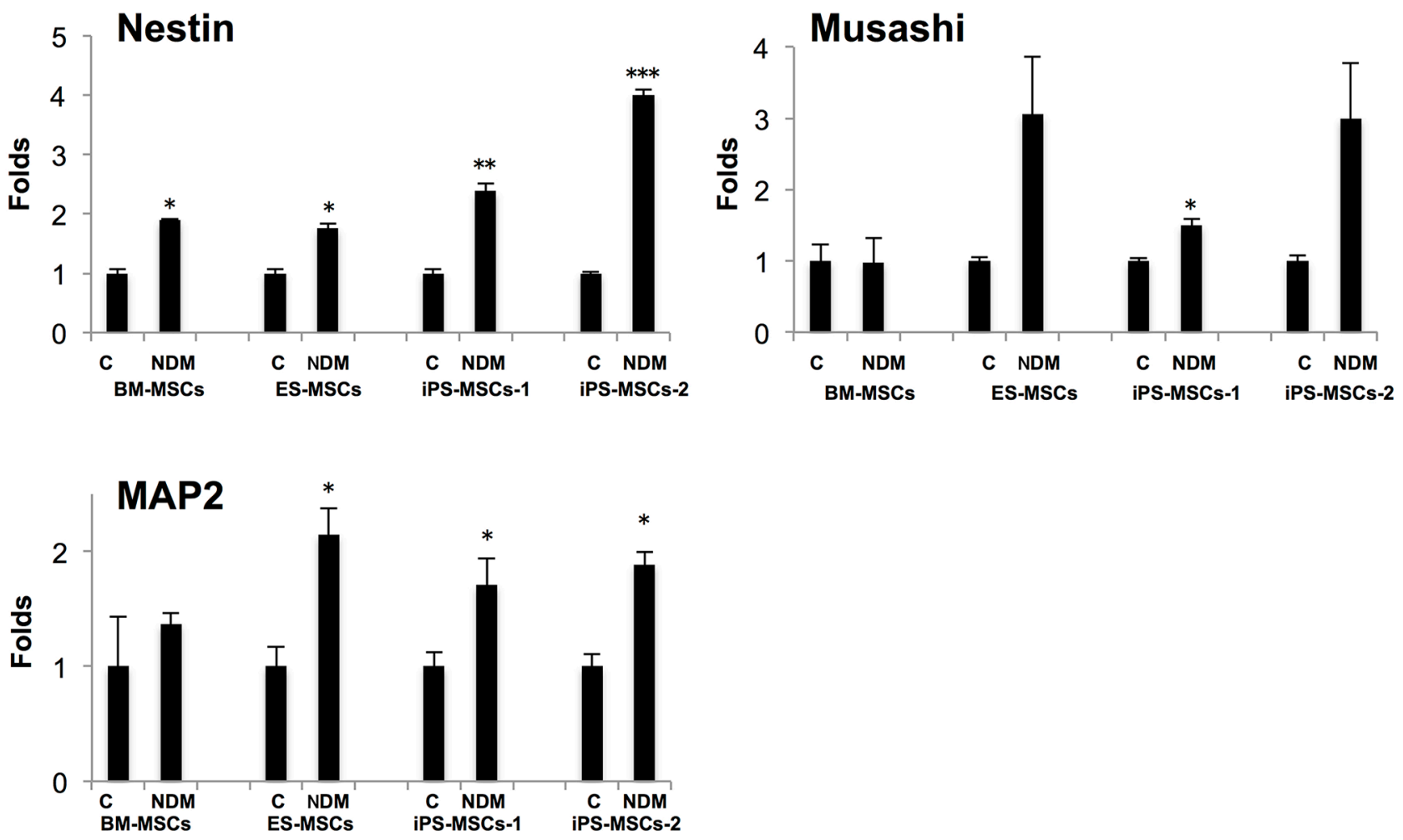

Figure 1: Human pluripotent stem cell-derived mesenchymal stem cells (PSC-MSCs) express higher level of neural stem cell-associated genes than bone marrow mesenchymal stem cells (BM-MSCs).(A) Gene expression of Nestin and Musashi in PSCMSCs(ES-MSCs, iPS-MSCs-1, andiPS-MSCs-2)andBM-MSCswasanalyzedbyreal-timePCR (qPCR). ${ }^{* * *} p<0.005$; comparedtoBM-MSCs. (B) Phase-contrast microscopy of BM-MSCs and various PSC-MSCs after being cultured 48 hours in neural differentiation medium (NDM; consist of RA $(0.5 \mu \mathrm{M})$ in serum-free medium). Arrow indicates dendrite-like processes. (C) Gene expression of Nestin, Musashi, and MAP2 in PSC-MSCs and BM-MSCs after culturing in control complete medium (C) and NDM for 48 hours as analyzed by qPCR. ${ }^{*} p<0.05$ and ${ }^{* *} p<0.01$, compared to Control. Error bars represent \pm SEM of three independent experiments. Scale bar: $20 \mu \mathrm{m}$. 
into committed cell types: neuron-restricted progenitors (NRPs) and glial-restricted progenitors (GRPs) [23, 24]. To explore whether PSC-MSCs can further differentiate into these more committed neural cells, we cultured cells in serum-free medium - a typical method to induce neural differentiation-with the addition of Y-27632 (SF+Y) [14]. Bright field microscopy showed that PSC-MSCs acquired more committed neural lineage morphology after $\mathrm{SF}+\mathrm{Y}$ treatment (Figure 3A). During the neural differentiation process from pluripotent cells into postmitotic neurons, the nucleus undergoes remodeling with concomitant decreases in size; this has been used as an indicator on the extent of neural differentiation [25]. We therefore measured the nuclear area of PSC-MSCs after various neural induction methods, and found that while Y-27632 in complete medium diminished the nuclear sizes of PSC-MSCs, the most significant reduction in nuclear size occurred when PSC-MSCs were cultured in $\mathrm{SF}+\mathrm{Y}$ especially for ES-MSCs (Figure 3B; average percentage of nuclear reduction: ES-MSCs from 100\% down to $33.3 \%$, iPS-MSCs-2 from down to $64.0 \%$, and
iPS-MSCs-3 down to $69.6 \%$ ). Furthermore, real-time PCR analysis for expression of $M A P 2$, which is a marker of NRPs [26], was also most significantly increased after $\mathrm{SF}+\mathrm{Y}$ treatment and most apparent in ES-MSCs as well (Figure 3C; average increases in ES-MSCs up to 3.8-fold, iPS-MSCs-1 up to 3.4-fold, iPS-MSCs-2 up to 4.8-fold). Thus, cytoskeletal manipulations in standard neurogenic differentiation conditions can further induce PSC-MSCs towards a more committed neural phenotype.

\section{PSC-MSCs express NRP-associated proteins after ROCK inhibition in SF conditions}

NRPs are positive for expression of $\beta$-III-tubulin, Doublecortin and NeuN, but negative for NSC markers such as Nestin [26, 27]. $\beta$-III-tubulin, also known as Tuj-1, is a neuron-specific tubulin whose expression is increased during neuronal development [28]. Doublecortin is a microtubule-associated protein, which is required for neuronal migration into the cerebral cortex [29]. NeuN (Neuronal Nuclei), a neuron-specific nuclear
A

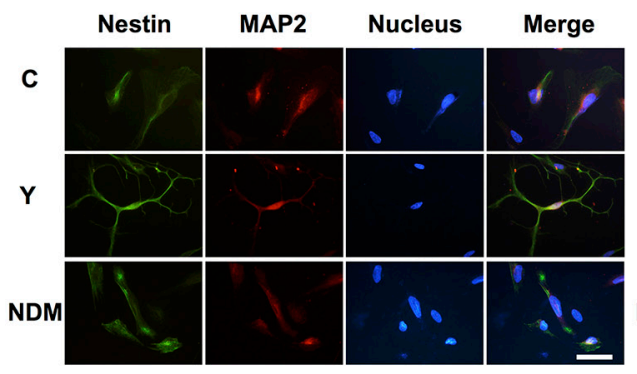

\section{iPS-MSCs-3}

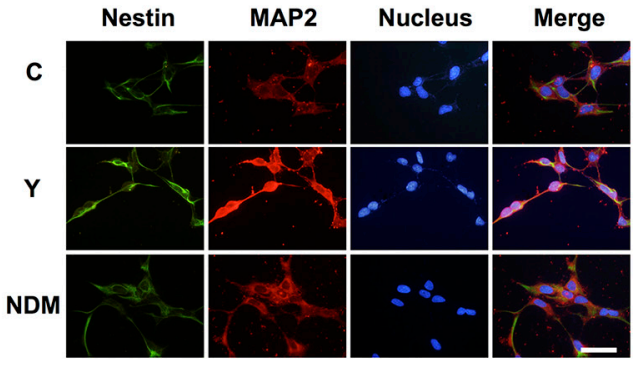

B

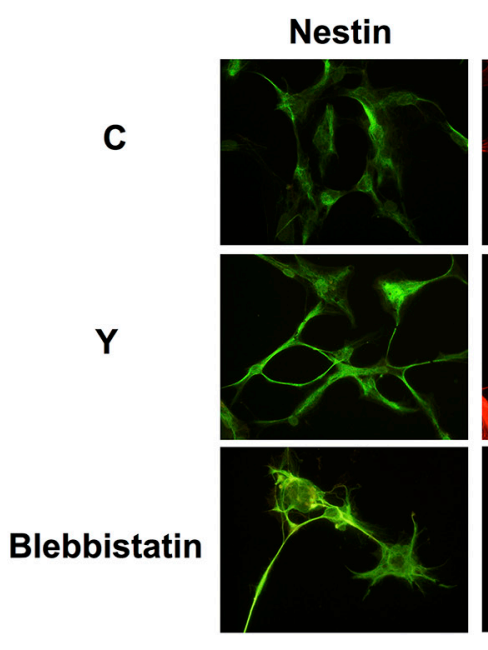

iPS-MSCs-3
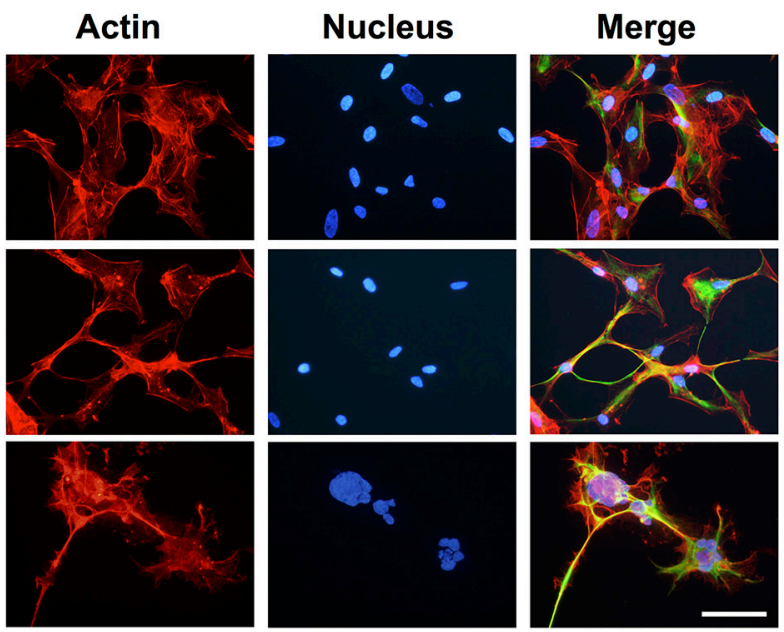

Figure 2: PSC-MSCs express early neural markes after RhoA kinase (ROCK) inhibition. (A) Immunofluorescent (IF) staining was performed to analyze for Nestin (green fluorescence) and MAP2 (red fluorescence) expression in PSC-MSCs after NDM or complete medium with ROCK inhibition using Y-27632 (Y). Hoechst 33342 was used to detect nuclei (blue). (B) Expression levels of Nestin (green fluorescence) and F-actin (red fluorescence) were analyzed by IF staining in PSC-MSCs after blebbistatin treatment. Scale bar: $20 \mu \mathrm{m}$. 
protein, regulates RNA splicing and marks postmitotic neuronal cells [30]. Using IF staining, we found that of all the differentiation conditions, $\mathrm{SF}+\mathrm{Y}$ resulted in the most apparent decreases in Nestin expression and concomitant increases in $\beta$-III-tubulin and Doublecortin expression (Figure 4A-4B). Interestingly, these changes in protein expression could only be seen in PSC-MSCs with committed neural cell morphology-cytoplasmic elongation, multiple dendritic process, and nuclear condensation-but not in PSC-MSCs that still retain undifferentiated, fibroblastic-typical MSC-cell morphology. Importantly, NeuN expression could also be detected in the nucleus of neural lineage-committed
PSC-MSCs, which is strongly suggestive of further commitment past the NSC stage and along the neural lineage for these MSCs (Figure 5A). Taken together, our data indicate that PSC-MSCs could differentiate into more committed neural cells through modulation of the ROCKmyosin II axis in a SF environment (Figure 5B).

\section{DISCUSSION}

Increasing evidence for MSC plasticity has brought hope for use of these versatile somatic stem cells for a multitude of clinical indications [6]. However, these rare progenitors are isolated from post-natal tissues, in which

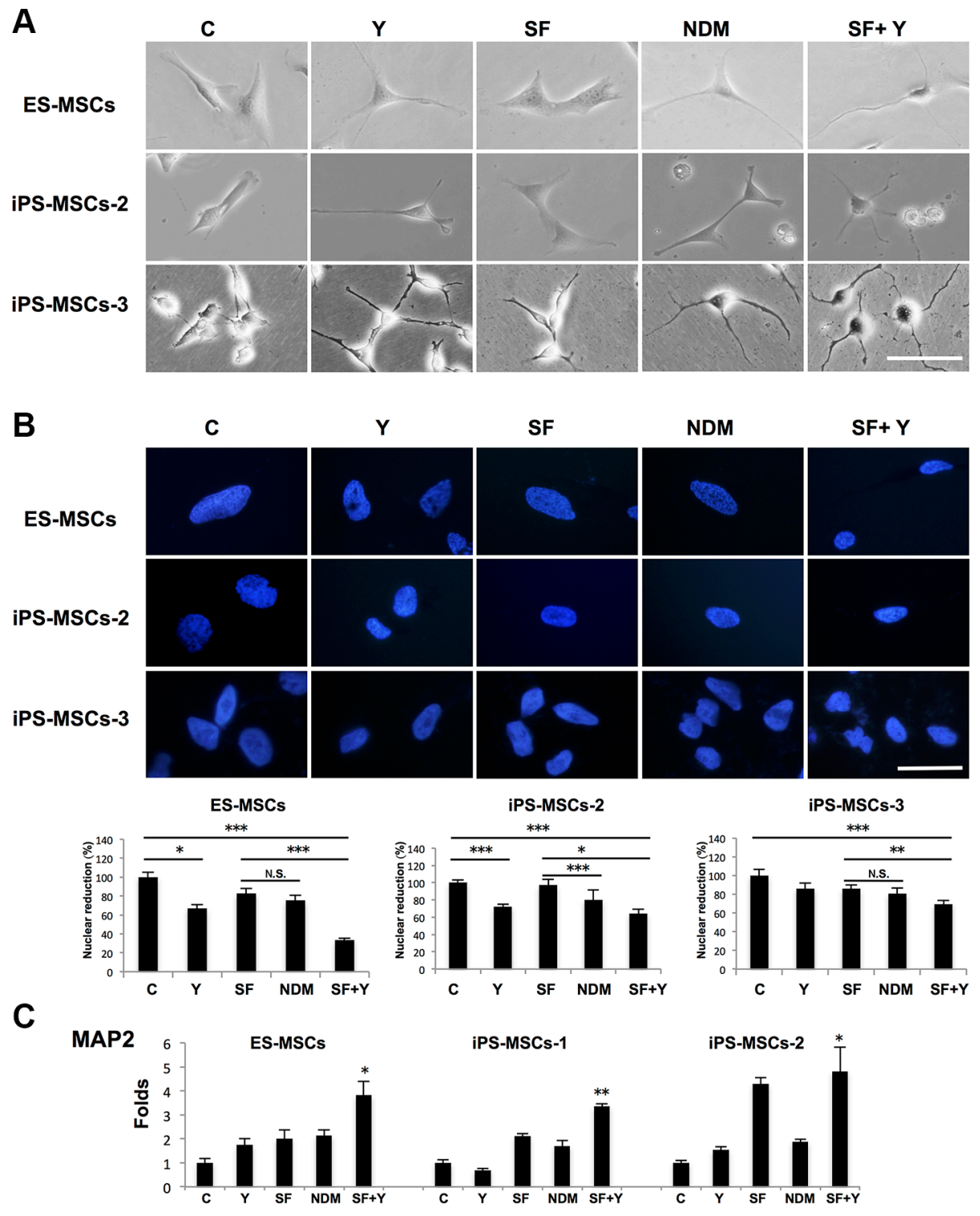

Figure 3: ROCK inhibition in serum-free (SF) conditions induce further neural lineage commitment of PSC-MSCs. (A) Phase-contrast microscopy of PSC-MSC cell morphology after ROCK inhibition with Y-27632 in complete medium (Y), NDM, and Y-27632 in serum-free medium (SF+Y). (B) Nuclear size of PSC-MSCs after culturing in Y, NDM, and SF+Y as quantified by Image J software. Nuclei were be detected by Hoechst 33342 (blue fluorescence). ${ }^{*} p<0.05 ; * * p<0.01 ; * * * p<0.005$; N.S., not significant. (C) Gene expression of MAP2 in PSC-MSCs after culturing in Y, NDM, and SF+Y as quantified as analyzed by qPCR. All results are shown as mean \pm SEM of three independent experiments. ${ }^{*} p<0.05$; ${ }^{*} p<0.01$; compared to control. Scale bar: $20 \mu \mathrm{m}$. 
senescence rapidly sets in during in vitro expansion for therapeutic use [7, 31]. Human PSC-MSCs including ES-MSCs and iPS-MSCs, are capable of multilineage paraxial mesodermal differentiation as well as immunomodulation, similar to adult BM-MSCs [12, 32]. Additionally, PSC-MSCs are highly proliferative and renewable without the worry of teratoma formation inherent to its parental cell type $[10,11]$. Our data demonstrates that PSC-MSCs have strong capacity to undergo neural differentiation, an extra-paraxial mesodermal lineage. Even in an undifferentiated state, PSC-MSCs expresses significantly higher levels of NSC markers such as Nestin and Musashi than adult BMMSCs. Moreover, in complete medium - the standard medium for culturing all types of MSCs-inhibition of the ROCK-myosin II axis in PSC-MSCs increases expression of Nestin. When ROCK inhibition is applied under more standard neurogenic differentiation conditions (SF medium), PSC-MSCs further differentiate into a more committed neural phenotype, as demonstrated by reduction of nuclear area, nuclear NeuN protein expression, and expression of more committed NRPproteins $\beta$-III-tubulin and Doublecortin. Our data therefore demonstrate that PSC-MSCs have the capacity to undergo significant neural lineage commitment. Ongoing studies are underway to ascertain the functionality of these PSCMSC-differentiated neural-like cells in in vivo disease models for future clinical applications.

Cytoskeletal rearrangement has increasingly been shown to be a critical component during neural development. The proteins of RhoA family are known to be involved in neuronal cytoplasmic extension and growth of dendritic processes seen during the development of the central nervous system $[16,17,33]$. At the stage of neurite initiation, F-actin polymerization in the growth cone results in the appearance of actin-rich lamellipodium [34]. MAP2 and Doublecortin, which we found to be increased in PSC-MSCs after suppression of the ROCK-myosin II axis, are involved in mediating neuronal morphological changes via regulation of the cytoskeleton $[35,36]$. Indeed, the severe neuronal phenotype found in Doublecortinknockout mice is due to actin dysregulation [37]. The
A

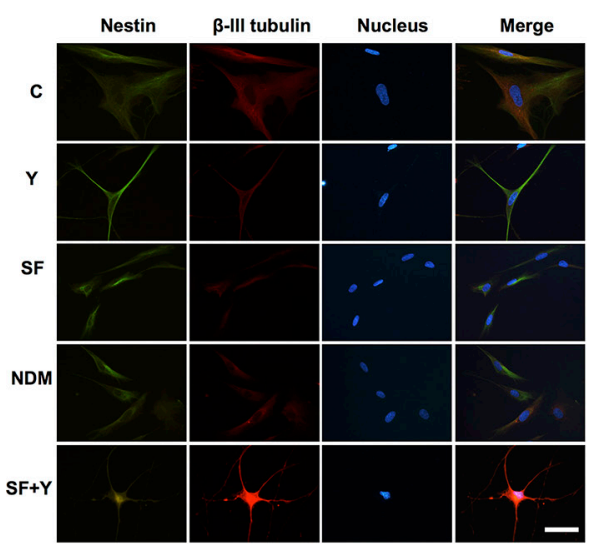

B

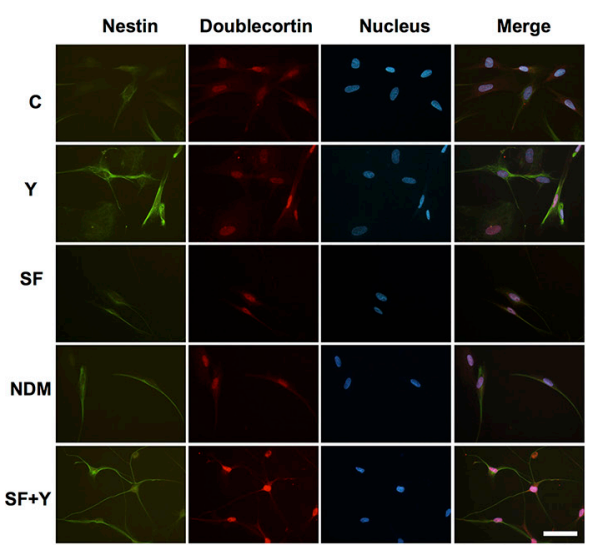

iPS-MSCs-3

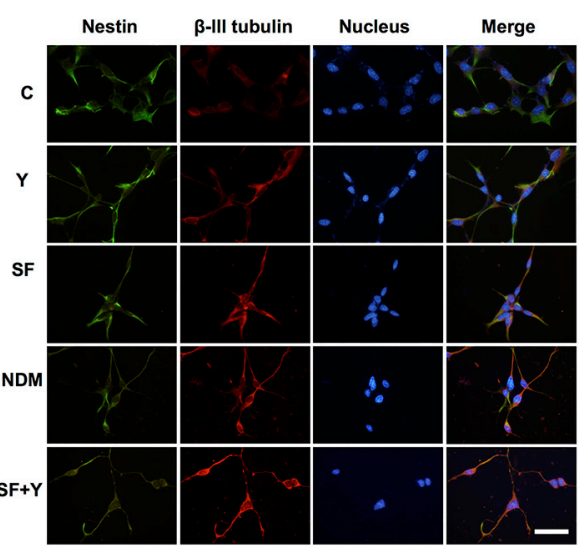

iPS-MSCs-3

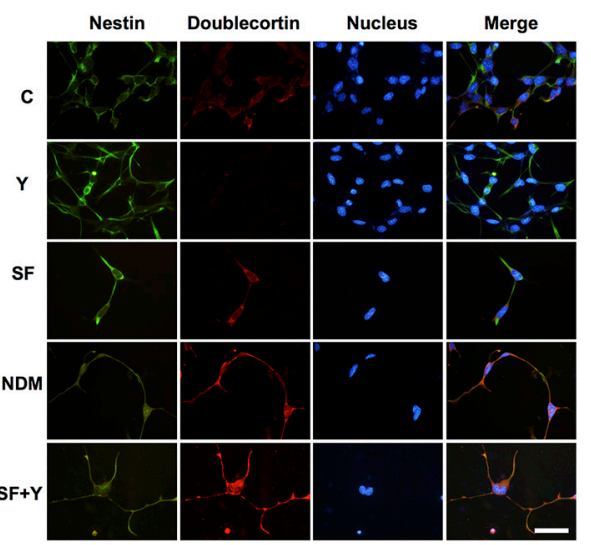

Figure 4: PSC-MSCs express committed neural lineage proteins after ROCK inhibition in SF conditions. Protein expression of (A) Nestin (green fluorescence) and $\beta$-III-tubulin (red fluorescence), or (B) Nestin (green fluorescence) and Doublecortin (red fluorescence) in PSC-MSCs after culturing in Y, NDM, and SF+Y as analyzed by IF staining. Nuclei were be detected by Hoechst 33342 (blue fluorescence). Scale bar: $20 \mu \mathrm{m}$. 
stage-specific importance of the cytoskeleton in neural development can be seen in our in vitro studies in which ROCK inhibition of PSC-MSCs in SF conditions - rather than with serum in complete medium - results acquisition of a more committed neural phenotype that is more profound than that induced with RA in SF medium. Our findings in this study are in line with other in vitro studies, in which inhibition of ROCK promotes neurite outgrowth in rat embryo hippocampal neurons and neuronal differentiation in mouse NSCs and ESCs [38, 39]. The importance of cell polarity during in vitro modeling of human PSC neural differentiation has also recently been shown in human iPSCs [40]. Moreover, recent reports also showed that ROCK inhibition could accelerate the process of direct reprogramming of fibroblasts into neurons [41]. These studies and ours further confirm the important role of the RhoA family and cytoskeletal rearrangement in MSC neural commitment, and demonstrate the utility of in vitro recapitulation of in vivo information.

While iPSCs and direct reprograming of fibroblasts can generate NRPs or other neural cells, these two cellular sources are not without its problems. For iPSCs, unless complete differentiation of all cells is achieved, teratoma formation is always a concern; the use of PSC-MSCs

A
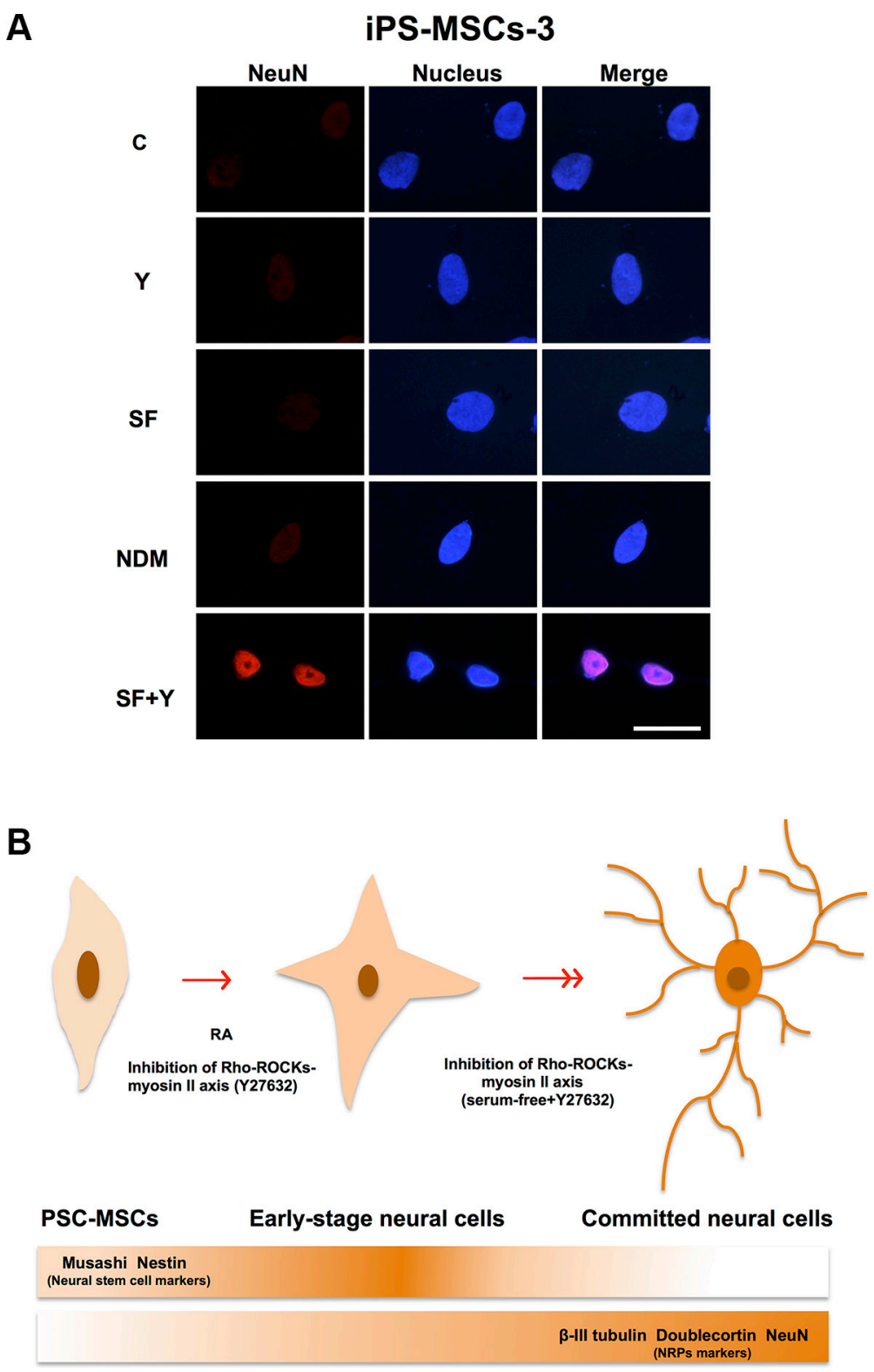

Figure 5: PSC-MSCs have differentiation capacity of neural lineage more than BM-MSCs. (A) IF staining for protein expression of NeuN (red fluorescence) PSC-MSCs cultured in SF+Y. Nuclei were be detected by Hoechst 33342 (blue fluorescence). Scale bar: $20 \mu \mathrm{m}$. (B) PSC-MSCs express higher baseline levels of NSC genes Nestin and Musashi than BM-MSCs. Culturing of PSC-MSCs in NDM or with inhibition of ROCK-myosin II axis leads to neural lineage commitment. When ROCK inhibition of PSC-MSCs is performed under SF conditions, these progenitors can further differentiate into more committed neural cells. 
abrogates this possibility. On the other hand, direct reprograming of fibroblasts to neural cells is highly inefficient as not to be clinically relevant currently [42]. In comparison to other adult/post-natal source MSCs such as BM-MSCs, PSC-MSCs possess a stronger capacity for neural differentiation; we speculate that this may be due to the early developmental stage of these MSCs being derived directly from PSCs, since it has been demonstrated that developmentally early stage MSCs isolated from fetal tissue can have broader differentiation capacity than adult BM-MSCs [43]. One possible mechanism accounting for these functional differences may be the higher expression levels of neural developmentally relevant genes such as Musashi in PSC-MSCs compared to BM-MSCs (Figure 1C). During neural development, Musashi, a translational regulator, represses $m-N u m b$ mRNA translation and activate Notch signaling [44]. This results in the maintenance of self-renewal and differentiation potential in NSCs [45]. Further studies would be necessary to confirm whether these early neural developmental specifiers are more active in PSC-MSCs compared to adult/post-natal MSCs.

\section{MATERIALS AND METHODS}

\section{Cell culture}

PSC-MSCs were derived and characterized as previously reported [10]. ES-MSCs were derived from HSF-6 (University of California, San Francisco) and H1 (Wisconsin Alumni Research Foundation) [46, 47]. iPS-MSCs were derived from iPS which were generated by two methods: (1) Sendai Virus induction (Sendai Reprogramming Kit; Invitrogen-Thermo Fisher Scientific, MA, USA) with all four Yamanaka factors into human peripheral blood mononuclear cells as per manufacturer's and published protocols [48] for iPS-MSC clones 1 and 2; and (2) lentiviral induction with Oct-4 and Sox-2 into human umbilical vein endothelial cells (Bioresource Collection and Research Center, Hsinchu, Taiwan) as previously reported [49] for iPS-MSC clone 3. BM-MSCs were obtained commercially (Promocell, Heidelberg, Germany) and cultured as previously described [4]. All MSCs were cultured with complete medium consisting of DMEM-low glucose supplemented with 1\% penicillin/ streptomycin, 1\% L-glutamine (all from Gibco-Thermo Fisher Scientific), and 10\% fetal bovine serum (FBS, selected lots from Hyclone-Thermo Fisher Scientific) and expanded as previously described [10].

\section{Differentiation studies}

PSC-MSCs and BM-MSCs were characterized for trilineage paraxial mesodermal differentiation capacity as previously described [10,50]. For neurogenic differentiation, cells were cultured on six-well plates $\left(5000 \mathrm{cells} / \mathrm{cm}^{2}\right)$ and treated with $0.5 \mu \mathrm{M}$ retinoic acid (RA; Sigma-Aldrich, St. Louis, MO, USA) for 48 hours in serum-free medium [51]. The ROCK inhibitor Y-27632 and blebbistatin $(10 \mu \mathrm{M}$, both from Sigma-Aldrich) was added to either complete medium or serum-free medium for 48 hours as indicated [15].

\section{Immunofluorescent (IF) staining}

IF staining was performed as previously reported [15]. Briefly, cells were fixed with $4 \%$ paraformaldehyde and permeabilized with $0.1 \%$ Triton-X 100 (Sigma-Aldrich) for 20 minutes. Cells were then blocked with 5\% BSA then incubated with primary antibodies against Nestin (1:100; mouse monoclonal, Millipore-Merck, Darmstadt, Germany), MAP2 (1:100; rabbit polyclonal, MilliporeMerck), $\beta$-III-tubulin (1:100; rabbit polyclonal, Abcam, Cambridge, MA, USA), Doublecortin (1:100; rabbit polyclonal, Abcam), or NeuN (1:100; mouse monoclonal, Millipore-Merck) at room temperature for two hours. Cells were washed with phosphate-buffered saline (PBS) and incubated with FITC-conjugated or PE-conjugated secondary antibodies (1:200; Santa Cruz Biotechnology, Santa Cruz, CA, USA) at room temperature for 2 hours. Nuclei were stained with Hoechst 33342 (Sigma-Aldrich). F-actin was detected by rhodamine-conjugated phalloidin (1:200; Invitrogen-Thermo Fisher Scientific). A fluorescence microscope was used for visualization (Olympus, Tokyo, Japan).

\section{Real-time quantitative polymerase chain reaction (real-time PCR)}

Real-time PCR was performed as previously reported [15]. Total RNAs were extracted with TRIzol Reagent (Invitrogen-Thermo Fisher Scientific) and cDNA was synthesized from RNA using RevertTra Ace cDNA Synthesis Kit (Toyobo, Osaka, Japan). Realtime PCR was performed by using Fast $\mathrm{SYBR}^{\circledR}$ Green Master Mix qPCR Kit on the ABI 7500 Real-Time PCR System (both from Applied Biosystems-Thermo Fisher Scientific). Relative gene expression levels were analyzed as indicated by the manufacturer. Primers used are shown in Table 1.

\section{Statistics}

All data were expressed as mean \pm SEM. Statistical significance between two samples was analyzed by the Student's $t$ test. ANOVA was used to analyze the significance between multiple samples. A value of $p<0.05$ was defined as statistical significance. 
Table 1: Primer list

\begin{tabular}{|c|c|c|}
\hline Gene & & Primer 5'-3' \\
\hline \multirow{2}{*}{ Nestin } & $\mathrm{F}$ & AACAGCGACGGAGGTCTCTA \\
\hline & $\mathrm{R}$ & TTCTCTTGTCCCGCAGACTT \\
\hline \multirow{2}{*}{ MAP2 } & $\mathrm{F}$ & CCAGGTGGCGGACGTGTGAA \\
\hline & $\mathrm{R}$ & GCCACGCTGGATCTGCCTGG \\
\hline \multirow{2}{*}{ Musashi-1 } & $\mathrm{F}$ & GTCTCGAGTCATGCCCTACG \\
\hline & $\mathrm{R}$ & GCAGTGAGAGGAATGGCTGT \\
\hline \multirow[b]{2}{*}{ GAPDH } & $\mathrm{F}$ & GTGGACCTGACCTGCCGTCT \\
\hline & $\mathrm{R}$ & GGAGGAGTGGGTGTCGCTGT \\
\hline
\end{tabular}

$\mathrm{F}$, forward primer; $\mathrm{R}$, reverse primer.

\section{CONCLUSIONS}

PSC-MSCs show potent capacity to differentiate into a committed neural phenotype with high expression of NSC genes at baseline. Cytoskeleton rearrangement through inhibition of the ROCK-myosin II axis in a neural differentiating environment can strongly induce PSCMSCs to acquire a committed neural cell phenotype, with reduction of nuclear size and upregulation of late neural lineage-specific markers including $\beta$-III-tubulin, Doublecortin, and nuclear NeuN. Our data gives support to the use of PSC-MSCs as a possible candidate progenitor cell in therapeutic application towards neuro-degenerative and related diseases, as well as highlight the importance of the cytoskeleton in neural differentiation.

\section{ACKNOWLEDGMENTS AND FUNDING}

This work was supported in part by funding from the NHRI (CS-105-PP-06 to B.L.Y.) and the Ministry of Science \& Technology (MOST) of Taiwan (MOST104-2321-B-400-021 to B.L.Y.). We also thank the Human Disease iPSC Service Consortium (MOST 1052325-B-001-009) of the National Research Program for Biopharmaceuticals (NRPB) of MOST for iPSC generation (iPS-MSC clones 1 and 2) and technical support.

\section{CONFLICTS OF INTEREST}

The authors declare no conflicts of interest.

\section{REFERENCES}

1. Lindvall O, Kokaia Z, Martinez-Serrano A. Stem cell therapy for human neurodegenerative disorders-how to make it work. Nat Med. 2004; 10:S42-50.

2. Reynolds BA, Rietze RL. Neural stem cells and neurospheres - re-evaluating the relationship. Nat Methods. $2005 ; 2: 333-336$.
3. Sanchez-Ramos JR. Neural cells derived from adult bone marrow and umbilical cord blood. J Neurosci Res. 2002; 69:880-893.

4. Pittenger MF, Mackay AM, Beck SC, Jaiswal RK, Douglas R, Mosca JD, Moorman MA, Simonetti DW, Craig S, Marshak DR. Multilineage potential of adult human mesenchymal stem cells. Science. 1999; 284:143-147.

5. Yen BL, Huang HI, Chien CC, Jui HY, Ko BS, Yao M, Shun CT, Yen ML, Lee MC, Chen YC. Isolation of multipotent cells from human term placenta. Stem Cells. 2005; 23:3-9.

6. Uccelli A, Laroni A, Freedman MS. Mesenchymal stem cells for the treatment of multiple sclerosis and other neurological diseases. Lancet Neurol. 2011; 10:649-656.

7. Ho PJ, Yen ML, Tang BC, Chen CT, Yen BL. H2O2 accumulation mediates differentiation capacity alteration, but not proliferative decline, in senescent human fetal mesenchymal stem cells. Antioxid Redox Signal. 2013; 18:1895-1905.

8. Lian Q, Zhang Y, Zhang J, Zhang HK, Wu X, Zhang Y, LamFF, Kang S, Xia JC, Lai WH, Au KW, Chow YY, Siu CW, et al. Functional mesenchymal stem cells derived from human induced pluripotent stem cells attenuate limb ischemia in mice. Circulation. 2010; 121:1113-1123.

9. Wang TS, Cheng PP, Qi ZQ. iPSC-MSCs and islet allograft tolerance. Oncotarget. 2015; 6:10669-10670. doi: 10.18632/oncotarget.4050.

10. Yen ML, Hou CH, Peng KY, Tseng PC, Jiang SS, Shun CT, Chen YC, Kuo ML. Efficient derivation and concise gene expression profiling of human embryonic stem cell-derived mesenchymal progenitors (EMPs). Cell Transplant. 2011; 20:1529-1545.

11. Zhao Q, Gregory CA, Lee RH, Reger RL, Qin L, Hai B, Park MS, Yoon N, Clough B, McNeill E, Prockop DJ, Liu F. MSCs derived from iPSCs with a modified protocol are tumor-tropic but have much less potential to promote tumors than bone marrow MSCs. Proc Natl Acad Sci U S A. 2015 ; 112:530-535. 
12. Yen BL, Chang CJ, Liu KJ, Chen YC, Hu HI, Bai CH, Yen ML. Brief report-human embryonic stem cellderived mesenchymal progenitors possess strong immunosuppressive effects toward natural killer cells as well as T lymphocytes. Stem Cells. 2009; 27:451-456.

13. Kogler G, Sensken S, Airey JA, Trapp T, Muschen M, Feldhahn N, Liedtke S, Sorg RV, Fischer J, Rosenbaum C, Greschat S, Knipper A, Bender J, et al. A new human somatic stem cell from placental cord blood with intrinsic pluripotent differentiation potential. J Exp. Med 2004; 200:123-135.

14. Fraichard A, Chassande O, Bilbaut G, Dehay C, Savatier P, Samarut J. In vitro differentiation of embryonic stem cells into glial cells and functional neurons. J Cell Sci. 1995; 108:3181-3188.

15. Wang CH, Wu CC, Hsu SH, Liou JY, Li YW, Wu KK, Lai YK, Yen BL. The role of RhoA kinase inhibition in human placenta-derived multipotent cells on neural phenotype and cell survival. Biomaterials. 2013; 34:3223-3230.

16. Groysman M, Shoval I, Kalcheim C. A negative modulatory role for rho and rho-associated kinase signaling in delamination of neural crest cells. Neural Dev. 2008; 3:27.

17. Luo L. Rho GTPases in neuronal morphogenesis. Nat Rev Neurosci 2000; 1:173-180.

18. Bauer HC, Tempfer H, Bernroider G, Bauer H. Neuronal stem cells in adults. Exp Gerontol. 2006; 41:111-116.

19. Dinsmore JH, Solomon F. Inhibition of MAP2 expression affects both morphological and cell division phenotypes of neuronal differentiation. Cell. 1991; 64:817-826.

20. Schmandke A, Schmandke A, Strittmatter SM. ROCK and Rho: biochemistry and neuronal functions of Rho-associated protein kinases. Neuroscientist. 2007; 13:454-469.

21. Limouze J, Straight AF, Mitchison T, Sellers JR. Specificity of blebbistatin, an inhibitor of myosin II. J Muscle Res Cell Motil. 2004; 25:337-341.

22. Kaech S, Parmar H, Roelandse M, Bornmann C, Matus A. Cytoskeletal microdifferentiation: a mechanism for organizing morphological plasticity in dendrites. Proc Natl Acad Sci U S A. 2001; 98:7086-7092.

23. Piper DR, Mujtaba T, Keyoung H, Roy NS, Goldman SA, Rao MS, Lucero MT. Identification and characterization of neuronal precursors and their progeny from human fetal tissue. J Neurosci Res. 2001; 66:356-368.

24. Mayer-Proschel M, Kalyani AJ, Mujtaba T, Rao MS. Isolation of lineage-restricted neuronal precursors from multipotent neuroepithelial stem cells. Neuro. 1997; 19:773-785.

25. Meshorer E, Yellajoshula D, George E, Scambler PJ, Brown DT, Misteli T. Hyperdynamic plasticity of chromatin proteins in pluripotent embryonic stem cells. Dev Cell. 2006; 10:105-116.

26. Mo Z, Moore AR, Filipovic R, Ogawa Y, Kazuhiro I, Antic SD, Zecevic N. Human cortical neurons originate from radial glia and neuron-restricted progenitors. J Neurosci. 2007; 27:4132-4145.

27. Cao QL, Howard RM, Dennison JB, Whittemore SR. Differentiation of engrafted neuronal-restricted precursor cells is inhibited in the traumatically injured spinal cord. Exp Neurol. 2002; 177:349-359.

28. Roskams AJ, Cai X, Ronnett GV. Expression of neuronspecific beta-III tubulin during olfactory neurogenesis in the embryonic and adult rat. Neuroscience. 1998; 83:191-200.

29. Gleeson JG, Lin PT, Flanagan LA, Walsh CA. Doublecortin is a microtubule-associated protein and is expressed widely by migrating neurons. Neuron. 1999; 23:257-271.

30. Kim KK, Adelstein RS, Kawamoto S. Identification of neuronal nuclei (NeuN) as Fox-3, a new member of the Fox-1 gene family of splicing factors. J Biol Chem. 2009; 284:31052-31061.

31. Wagner W, Horn P, Castoldi M, Diehlmann A, Bork S, Saffrich R, Benes V, Blake J, Pfister S, Eckstein V, Ho AD. Replicative senescence of mesenchymal stem cells: a continuous and organized process. PLoS One. 2008; 3:e2213.

32. Giuliani M, Oudrhiri N, Noman ZM, Vernochet A, Chouaib S, Azzarone B, Durrbach A, Bennaceur-Griscelli A. Human mesenchymal stem cells derived from induced pluripotent stem cells down-regulate NK-cell cytolytic machinery. Blood. 2011; 118:3254-3262.

33. Compagnucci C, Piemonte F, Sferra A, Piermarini E, Bertini E. The cytoskeletal arrangements necessary to neurogenesis. Oncotarget. 2016; 7:19414-29. doi: 10.18632/oncotarget.6838.

34. Dehmelt L, Halpain S. Actin and microtubules in neurite initiation: are MAPs the missing link? J Neurobiol. 2004; 58:18-33.

35. Francis F, Koulakoff A, Boucher D, Chafey P, Schaar B, Vinet MC, Friocourt G, McDonnell N, Reiner O, Kahn A, McConnell SK, Berwald-Netter Y, Denoulet P, et al. Doublecortin is a developmentally regulated, microtubuleassociated protein expressed in migrating and differentiating neurons. Neuron. 1999; 23:247-256.

36. Tint I, Jean D, Baas PW, Black MM. Doublecortin associates with microtubules preferentially in regions of the axon displaying actin-rich protrusive structures. J Neurosci. 2009; 29:10995-11010.

37. Fu X, Brown KJ, Yap CC, Winckler B, Jaiswal JK, Liu JS. Doublecortin (Dcx) family proteins regulate filamentous actin structure in developing neurons. J Neurosci. 2013; 33:709-721.

38. Da Silva JS, Medina M, Zuliani C, Di Nardo A, Witke W, Dotti CG. RhoA/ROCK regulation of neuritogenesis via profilin IIa-mediated control of actin stability. J Cell Biol. $2003 ; 162: 1267-1279$.

39. Li X, Zuo X, Jing J, Ma Y, Wang J, Liu D, Zhu J, Du X, Xiong L, Du Y, Xu J, Xiao X, Wang J, et al. SmallMolecule-Driven Direct Reprogramming of Mouse Fibroblasts into Functional Neurons. Cell Stem Cell. 2015; 17:195-203. 
40. Compagnucci C, Petrini S, Higuraschi N, Trivisano M, Specchio N, Hirose S, Bertini E, Terracciano A. Characterizing PCDH19 in human induced pluripotent stem cells (iPSCs) and iPSC-derived developing neurons:emerging role of a protein involved in controlling polarity during neurogenesis. Oncotarget. 2015; 6:26804-26813. doi: 10.18632/oncotarget.5757.

41. Chang TC, Chen YC, Yang MH, Chen CH, Hsing EW, Ko BS, Liou JY, Wu KK. Rho kinases regulate the renewal and neural differentiation of embryonic stem cells in a cell plating density-dependent manner. PLoS One. 2010; 5:e9187.

42. Broccoli V, Rubio A, Taverna S, Yekhlef L. Overcoming the hurdles for a reproducible generation of human functionally mature reprogrammed neurons. Exp Biol Med (Maywood). 2015; 240:787-794.

43. Barberi T, Willis LM, Socci ND, Studer L. Derivation of multipotent mesenchymal precursors from human embryonic stem cells. PLoS Med. 2005; 2:e161.

44. Imai $\mathrm{T}$, Tokunaga $\mathrm{A}$, Yoshida $\mathrm{T}$, Hashimoto M, Mikoshiba $\mathrm{K}$, Weinmaster G, Nakafuku M, Okano H. The neural RNA-binding protein Musashil translationally regulates mammalian numb gene expression by interacting with its mRNA. Mol Cell Biol. 2001; 21:3888-3900.

45. Okano H, Kawahara H, Toriya M, Nakao K, Shibata S, Imai T. Function of RNA-binding protein Musashi-1 in stem cells. Exp Cell Res. 2005; 306:349-356.
46. Abeyta MJ, Clark AT, Rodriguez RT, Bodnar MS, Pera RA, Firpo MT. Unique gene expression signatures of independently-derived human embryonic stem cell lines. Hum Mol Genet. 2004; 13:601-608.

47. Cheng L, Hammond H, Ye Z, Zhan X, Dravid G. Human adult marrow cells support prolonged expansion of human embryonic stem cells in culture. Stem Cells. 2003; 21:131-142.

48. Seki T, Yuasa S, Oda M, Egashira T, Yae K, Kusumoto D, Nakata H, Tohyama S, Hashimoto H, Kodaira M, Okada Y, Seimiya H, Fusaki N, et al. Generation of induced pluripotent stem cells from human terminally differentiated circulating T cells. Cell Stem Cell. 2010; 7:11-14.

49. Ho PJ, Yen ML, Lin JD, Chen LS, Hu HI, Yeh CK, Peng CY, Lin CY, Yet SF, Yen BL. Endogenous KLF4 expression in human fetal endothelial cells allows for reprogramming to pluripotency with just OCT3/4 and SOX2 - brief report. Arterioscler Thromb Vasc Biol. 2010; 30:1905-1907.

50. Yen BL, Yen ML, Hsu PJ, Liu KJ, Wang CJ, Bai CH, Sytwu HK. Multipotent human mesenchymal stromal cells mediate expansion of myeloid-derived suppressor cells via hepatocyte growth factor/c-met and STAT3. Stem Cell Reports. 2013; 1:139-151.

51. Kim M, Habiba A, Doherty JM, Mills JC, Mercer RW, Huettner JE. Regulation of mouse embryonic stem cell neural differentiation by retinoic acid. Dev Biol. 2009; $328: 456-471$. 